\title{
Efficient realisation of discrete Fourier transforms using the recursive discrete Hartley transform
}

W.C. Siu

K.L. Wong

Indexing terms: Algorithms, Signal processing

Abstract: In the paper, we present the results of our study using a recursive discrete Hartley transform technique to compute discrete Fourier transforms. We also introduce an improved in-place and in-order prime-factor mapping to effectively realise composite-length DFTs. In using these new techniques, the speed of computation is comparable to that of the Winograd Fourier transform algorithm (WFTA), whereas the program size of the present approach is much smaller than that of the WFTA. This approach is most suitable for the cases where there are restrictions on program lengths.

\section{Introduction}

The discrete Fourier transform (DFT) is one of the most important tools in modern digital signal processing. Much research effort has been dedicated to its efficient realisations. The fast Fourier transform (FFT) [1] proposed by Cooley and Tukey reduces the number of multiplications from $N^{2}$ to $(N / 2)\left(\log _{2} N\right)$, where $N$ is the transform length. Good [2] showed that a single dimension DFT can be converted to a multidimensional form if the transform length is composed of relatively prime factors. Winograd [3] and Kolba and Parks [4] made use of Rader's theorem [5] to construct efficient algorithms of this class. Compared with FFT, these latter approaches further reduce the number of multiplications by one-half to two-thirds. Siu and Constantinides [6], made use of Rader's algorithm to propose the very fast discrete Fourier transform (VFDFT) based on the number theoretic transform (NTT) which achieved having only one multiplication per point.

Recently, Siu [7] proposed a nesting algorithm for the VFDFT which recursively decomposed the discrete Fourier transform into short modules that just required two very efficient primary DFT modules of length 2 and 4. This paper extends this work by developing another efficient algorithm making use of the discrete Hartley transform (DHT) [8]. The present work solves the problem of a prime-factor mapping for DHT which is an essential step for the recursive decomposition. Sorensen et al. [9] proposed a modified prime-factor mapping technique based upon Good's algorithm [2] to construct

Paper 6710E (C1, C2), first received 26th July 1988 and in revised form 6th February 1989

The authors are with the Department of Electronic Engineering, Hong Kong Polytechnic, Hung Hom, Kowloon, Hong Kong composite-length DHT using prime-length modules. The control structure of this approach is complicated since data from different short transforms within the same stage are coupled by some extra additions. In this paper, we use a simpler but more general approach in which a recursive DHT technique is used to formulate a fast algorithm for the computation of DFT. An improved in-place in-order prime-factor mapping is also developed for the construction of long composite length transforms.

\section{Recursive discrete Hartley transform}

The discrete Hartley transform of a real data sequence $\{r(n): n=0,1, \ldots, N-1\}$ is defined as

$$
Q(k)=\sum_{n=0}^{N-1} r(n) \operatorname{cas}(2 \pi n k / N)
$$

where $\operatorname{cas} \beta=\cos \beta+\sin \beta$.

Note that the DHT is structurally very similar to the DFT. Most importantly, it retains the cyclic property which was made used by Rader [5] and Siu and Constantinides [6] to convert prime-length DFTs into cyclic correlation and cyclic convolution forms, respectively. This enables the same conversion to be made on the DHT. More precisely, if $N=P$, where $P$ is a prime number with a primitive root equal to $g$, eqn. 1 can be written as

$$
Q(0)=\sum_{n=0}^{P-1} r(n)
$$

and

$$
Q_{k}=R_{k}+r(0)
$$

where

$$
\begin{aligned}
Q_{k} & =Q\left(\left\langle g^{k-1}\right\rangle_{P}\right) \\
R_{k} & =\sum_{n=0}^{P-2} r_{n} A_{k-n} \\
A_{n} & =\operatorname{cas}\left(2 \pi\left\langle g^{n-2}\right\rangle_{P} / P\right) \\
r_{n} & =r\left(\left\langle g^{-(n+1)}\right\rangle_{P}\right)
\end{aligned}
$$

$$
\text { for } k, n=0,1, \ldots, P-2
$$

The expression $\langle C\rangle_{P}$ means the residue of the number $C$ modulo $P$. Eqn. 5 is a length-( $P-1)$ cyclic convolution. A previous effort [7] made use of a slightly different permutation to convert a prime-length DFT into this form and evaluated the convolution based on length- $(P-1)$ DFTs. As the DHT also possesses convolution properties [8], eqn. 5 can be realised using length- $(P-1)$ DHTs. 
That is,

$$
\begin{aligned}
R_{k}^{\prime}= & \sum_{n=0}^{P-2} r_{n} \operatorname{cas}(2 \pi n k /(P-1)) \\
A_{k}^{\prime}= & \sum_{n=0}^{P-2} A_{n} \operatorname{cas}(2 \pi n k /(P-1)) \\
A_{e k}^{\prime}= & \left(A_{k}^{\prime}+A_{-k}^{\prime}\right) / 2 \\
A_{o k}^{\prime}= & \left(A_{k}^{\prime}-A_{-k}^{\prime}\right) / 2 \\
R_{k}= & \frac{1}{P-1} \sum_{n=0}^{P-2}\left(R_{n}^{\prime} A_{e n}^{\prime}+R_{-n}^{\prime} A_{o n}^{\prime}\right) \\
& \times \operatorname{cas}(2 \pi n k /(P-1))
\end{aligned}
$$

Note that $A_{e k}^{\prime}$ and $A_{o k}^{\prime}$ are even and odd parts of $A_{k}^{\prime}$, respectively. Hence, we have succeeded in decomposing a length- $P$ DHT into two length- $(P-1)$ DHTs. This approach is particularly attractive for transforms of length $3(3-1=2)$ and length $5(5-1=4)$, since DHTs of lengths 2 and 4 required no multiplications.

For longer prime lengths, the present approach will have difficulty in recursively decomposing [7] into the two efficient transform modules since, in general, a composite-length DHT cannot be simply decomposed into shorter DHT modules using the prime-factor mapping technique [9]. Let us elaborate this point by considering a composite number $D=S \times T$ where $S$ and $T$ are relatively prime. We can convert a length- $D$ DHT

$$
\begin{aligned}
& Q(k)=\sum_{n=0}^{D-1} r(n) \operatorname{cas}(2 \pi n k / D) \\
& \qquad \text { for } n, k=0,1, \ldots, D-1
\end{aligned}
$$

into a multidimensional form

$$
\begin{aligned}
Q\left(k_{1}, k_{2}\right)=\sum_{n_{2}=0}^{T-1} \sum_{n_{1}=0}^{S-1} r\left(n_{1}, n_{2}\right) & \\
& \times \operatorname{cas}\left(2 \pi\left(\frac{n_{1} k_{1}}{S}+\frac{n_{2} k_{2}}{T}\right)\right)
\end{aligned}
$$

where

$$
\begin{aligned}
& n=\left\langle T n_{1}+S n_{2}\right\rangle_{D} \\
& k=\left\langle T T^{-1} k_{1}+S S^{-1} k_{2}\right\rangle_{D}
\end{aligned}
$$

with

$$
\begin{aligned}
T T^{-1} & =1 \bmod S \\
S S^{-1} & =1 \bmod T \\
\text { for } n_{1}, k_{1} & =0,1, \ldots, S-1 \text { and } \\
n_{2}, k_{2} & =0,1, \ldots, T-1 .
\end{aligned}
$$

It is not always true that cas $(U+V)$ be equal to the product of cas $U$ and cas $V$. Hence, eqn. 14 cannot be directly decomposed into short transforms of lengths $S$ and $T$, respectively. However, consider a special case when one of the factors, say $T$, is equal to 2 . Eqn. 14 becomes

$$
\begin{aligned}
& Q\left(k_{1}, 0\right)=Q_{S}\left(k_{1}, 0\right)+Q_{S}\left(k_{1}, 1\right) \\
& Q\left(k_{1}, 1\right)=Q_{S}\left(k_{1}, 0\right)-Q_{S}\left(k_{1}, 1\right)
\end{aligned}
$$

where

$$
Q_{S}\left(k_{1}, n_{2}\right)=\sum_{n_{1}=0}^{S-1} r\left(n_{1}, n_{2}\right) \operatorname{cas}\left(2 \pi n_{1} k_{1} / S\right)
$$

Recombining eqns. 17 and 18 , we have

$$
\begin{aligned}
Q\left(k_{1}, k_{2}\right)= & \sum_{n_{2}=0}^{1} Q_{S}\left(k_{1}, n_{2}\right) \operatorname{cas}\left(2 \pi n_{2} k_{2} / 2\right) \\
= & \sum_{n_{2}=0}^{1} \sum_{n_{1}=0}^{s-1} r\left(n_{1}, n_{2}\right) \\
& \times \operatorname{cas}\left(\frac{2 \pi n_{1} k_{1}}{T}\right) \operatorname{cas}\left(\frac{2 \pi n_{2} k_{2}}{2}\right)
\end{aligned}
$$

Hence, we have shown that the prime-factor mapping technique is applicable to DHT in this special case, i.e. for the transform length $D=S \times 2$, where $S$ is an odd integer. This important fact enables us to recursively construct longer prime-length DHTs based on the efficient length-2 and 4 DHT modules. For example, length-7 $(7-1=3 \times 2)$ and length-11 (11-1=5×2) DHTs can be realised by using this technique. Due to the recursive nature of this algorithm, we call it the recursive DHT (RDHT).

\section{Discrete Fourier transform based on RDHT}

Given the DHTs of the real and the imaginary parts of a complex sequence, it is easy to convert them into the DFT of the sequence. The DFT of a complex sequence $\{x(n): n=0,1, \ldots, N-1\}$ is defined as

$$
Y(k)=\sum_{n=0}^{N-1} x(n) W^{n k}
$$

where

$$
W=e^{-j 2 \pi / N}=\cos (2 \pi / N)+j \sin (2 \pi / N)
$$

The DFT of eqn. 21 can be evaluated by the following formulations using the DHT:

$$
\begin{aligned}
& \operatorname{Re}\{Y(k)\}=(X R(k)+X R(-k)-X I(k)+X I(-k)) / 2 \\
& \operatorname{Im}\{Y(k)\}=(X R(k)-X R(-k) \\
&+X I(k)+X I(-k)) / 2
\end{aligned}
$$

where

$$
X R(k)=\operatorname{DHT}\{\operatorname{Re}\{x(n)\}\}
$$

and

$$
X I(k)=D H T\{\operatorname{Im}\{x(n)\}\}
$$

The operators $\operatorname{Re}\{\cdot\}$ and $\operatorname{Im}\{\cdot\}$ denote the real and imaginary parts of a complex number, respectively. Using the RDHT algorithm developed above, we can obtain efficient prime-length DFT algorithms with eqns. 22-25. Since all odd prime-length transform modules are realised with a standard sequence of operations, their implementations are greatly simplified by this highly regular control structure. It is shown in a later part of the paper that the factor $(1-P)^{-1}$ in eqn. 12 can be merged with other multiplication constants so that the IDHT can be performed with a DHT module, since their transform kernels are identical. Moreover, the recursive construction of large transform modules based on only two primitive modules, namely, DHTs of length 2 and 4, reduces much of the design effort. Separate treatment of the real and the imaginary parts further improves the algorithms in this aspect. In terms of software realisation, the new approach results in high productivity and has very short program lengths. 
In actual implementation, the efficiency can further be improved by noting the following properties:

(1) Precompute constants: the sequences $A_{e k}^{\prime}$ and $A_{o k}^{\prime}$ are fixed for every short prime-length DHT. They can be precomputed and stored as constants in actual realisations. This arrangement can eliminate run-time evaluation of eqns. 9-11. Furthermore, these constants can be merged with the factors $1 /(P-1)$ in eqn. 12 and $1 / 2$ in eqns. 22 and 23 , so that no actual division is needed.

(2) Evaluation of DC term: the application of eqn. 2 to the real and the imaginary parts of the input sequence separately will give the DC term of the DFT at the expense of $2(N-1)$ additions. However, from eqns. 7 and

$$
R_{0}^{\prime}=\sum_{n=0}^{P-2} r_{n}=\sum_{n=1}^{P-1} r(n)
$$

hence

$$
Q(0)=r(0)+R_{0}^{\prime}
$$

Since $R_{0}^{\prime}$ is already evaluated in eqn. 8 , only one extra addition is needed to obtain $Q(0)$. That is, $2(N-2)$ additions are eliminated for the computation of the DC term in a complex DFT.

(3) Computation of frequency components: eqn. 3 requires one addition for every $Q_{k}$. Note that $R_{k}$ in eqn. 3 is itself obtained from an IDHT in eqn. 12. If we define

$$
\begin{aligned}
& Q_{0}^{\prime}=R_{0}^{\prime} A_{k}^{\prime}+r(0) / 2 \\
& Q_{k}^{\prime}=R_{k}^{\prime} A_{e k}^{\prime}+R_{-k}^{\prime} A_{o k}^{\prime}
\end{aligned}
$$

$$
\text { for } k=1,2, \ldots, P-2
$$

it can be easily shown that the sequence $\left\{Q_{k}\right\}$ can be obtained by directly applying IDHT to $\left\{Q_{k}^{\prime}\right\}$. Note also that we divide $r(0)$ by 2 since the precomputed constants are already scaled by the factor $1 / 2(P-1)$ described above. That is, the $(N-1)$ additions in eqn. 3 are replaced by only one addition and one shift operation in eqn. $28 a$. The additions in eqn. $28 b$ are included in the original algorithm. For a complex DFT, this observation eliminates another $2(N-2)$ additions.

Fig. 1 shows the sequence of operations involved in the RDHT algorithm which is common to all prime length transforms. Details of operations within each block can be systematically derived as described previously. The simple interfaces between the blocks contribute to the regular control structure of the algorithm.

\section{Examples}

To illustrate the idea, let us consider the DFT of a complex data sequence $\{x(0), x(1), x(2), x(3), x(4)\}$. In this case, the transform length $N=P=5$ and its primitive root $g=2$. Define $x r(n)$ and $x i(n)$ to be the real and imaginary parts of $x(n)$, i.e. $x(n)=x r(n)+j x i(n)$, for $n=0,1$, $2,3,4$. Consider the sequence $\{x r(n): n=0,1,2,3,4\}$. To apply RDHT, we have to map the sequence using the primitive root permutation described in eqn. 7 to

$$
\begin{aligned}
\left\{r_{n}: n=0,1,2,3\right\} & =\left\{\operatorname{xr}\left(\left\langle g^{-(n+1)}\right\rangle_{5}\right): 0,1,2,3\right\} \\
& =\{\operatorname{xr}(3), \operatorname{xr}(4), \operatorname{xr}(2), \operatorname{xr}(1)\}
\end{aligned}
$$

We have to convolve this sequence with the sequence $\left\{A_{n}: n=0,1,2,3\right\}$, that is,

$$
\begin{aligned}
& \{\operatorname{xr}(3), \operatorname{xr}(4), \operatorname{xr}(2), \operatorname{xr}(1)\} \otimes \\
& \quad\{\operatorname{cas}(4(2 \pi / 6)), \operatorname{cas}(3(2 \pi / 6)), \operatorname{cas}(2 \pi / 6), \operatorname{cas}(2(2 \pi / 6))\}
\end{aligned}
$$

To obtain $\left\{R_{k}^{\prime}\right\}$, the sequence $\left\{r_{n}\right\}$ is transformed by a length-4 DHT, which requires no nontrivial multiplication. $X R(0)$, as defined in eqn. 24 , can be obtained by adding $R_{0}^{\prime}$, the DC term of this length-4 DHT, to $\operatorname{xr}(0)$. On the other hand, the sequence $\left\{A_{n}^{\prime}\right\}$ is precomputed and merged with the constant factors mentioned previously. We have

$$
\begin{aligned}
{\left[\begin{array}{l}
A_{0}^{\prime} \\
A_{1}^{\prime} \\
A_{2}^{\prime} \\
A_{3}^{\prime}
\end{array}\right] } & =\left[\begin{array}{l}
A_{e 0}^{\prime} \\
A_{e 1}^{\prime} \\
A_{e 2}^{\prime} \\
A_{e 3}^{\prime}
\end{array}\right]+\left[\begin{array}{l}
A_{o 0}^{\prime} \\
A_{o 1}^{\prime} \\
A_{o 2}^{\prime} \\
A_{o 3}^{\prime}
\end{array}\right] \\
& =\left[\begin{array}{r}
-0.125 \\
-0.2378 \\
0.2995 \\
-0.2378
\end{array}\right]+\left[\begin{array}{c}
0.0 \\
-0.1469 \\
0.0 \\
0.1469
\end{array}\right]
\end{aligned}
$$

Note that $A_{e n}^{\prime}=A_{e(-n)}^{\prime}$ and $A_{o n}^{\prime}=-A_{o(-n)}^{\prime}$. These properties can be used to reduce the storage required by the precomputed constants. Also note that $A_{0}^{\prime}, A_{1}^{\prime}, A_{2}^{\prime}$ and $A_{3}^{\prime}$ are scaled by $1 / 8(=1 / 2 \times 1 / 4)$. The sequence $\left\{Q_{k}^{\prime}\right\}$ is evaluated as shown in eqns. $28 a$ and $28 b$. As the factor $1 /(P-1)$, i.e. $1 / 4$, has already been merged with $\left\{A_{n}^{\prime}\right.$ : $n=0,1,2,3\},\{X R(k): k=1,2,3,4\}$ can then be obtained by applying a length-4 DHT to $\left\{Q_{k}^{\prime}\right\}$ followed by a primitive root permutation. Only one transform module is needed for both forward and inverse transforms. Applying the same set of operations to $\{x i(k)\}$ will give $\{X I(k): k=0,1,2,3,4\}$. $\{Y(k): k=0,1,2,3,4\}$ can then be calculated, as described in eqns. 22 and 23 , without the scaling factor $1 / 2$ as it has already been merged with $\left\{A_{n}^{\prime}\right\}$.

Similarly, we can construct RDHT-DFTs of other prime lengths. For longer lengths, however, an additional effort has to be paid to take care of the prime factor mapping decomposition of the length- $(P-1)$ DHT. Care must also be taken over the fact that the multiplication constants of the shorter prime-length RDHTs will scale each datum of the length-( $(P-1)$ DHT differently. For example, the data in a length- 11 transform must first be rearranged according to the primitive-root permutation into a convolution form. They are then further mapped into a two-dimensional form so that the convolution can be realised using DHTs of length 2 and 5. The length- 5 DHT module just developed can then be used in the

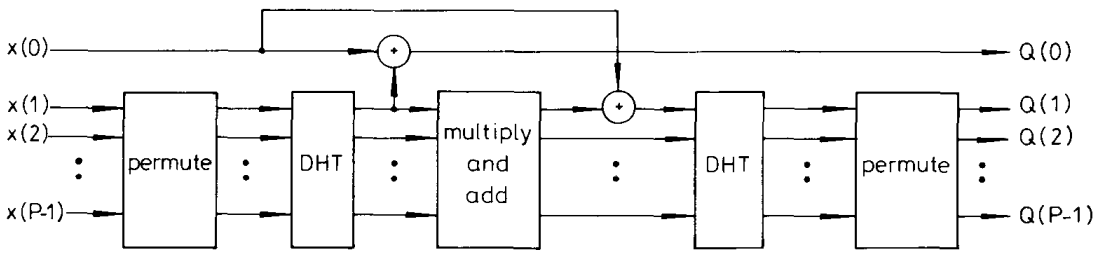

Fig. 1 Recursive discrete Hartley transform 
present case to reduce programming effort and to shorten code length. We have to recall that a scaling factor of $1 / 2$ has to be included in this computation to eliminate the divisions in eqns. 22 and 23 . As this module is used in both the forward and the inverse transforms, involved data will be so scaled twice. Note also that the DC term in each length- 5 module is not scaled, whereas the corresponding terms in the length-11 transform have to be scaled. These can be compensated by suitably adjusting the multiplication constants in the length- 11 module, as found in the program listing at the end of the paper.

\section{$5 \quad$ Improved prime factor mapping}

The RDHT algorithm will be increasingly complicated for long transform lengths. We can resolve this problem by constructing a long composite-length DFT from the short prime-length transforms using the prime-factor mapping [2]. An in-place, in-order mapping scheme has been proposed by Arambepola [10]. This method is to map the transform indexes into two-dimensional forms by the following equations:

$$
\begin{aligned}
& n=S_{1}\left(n^{\prime}, n_{r}\right)=\left\langle N_{r} n^{\prime}+N^{\prime} n_{r}\right\rangle_{N} \\
& k=S_{2}\left(n^{\prime}, k_{r}\right)=\left\langle N_{r} n^{\prime}+N^{\prime} N^{\prime-1} k_{r}\right\rangle_{N}
\end{aligned}
$$

where $N=N_{r} N^{\prime}$ is the composite transform length; $N_{r}$ is the short length currently used; $N^{\prime}=N / N_{r}$ and $N^{\prime-1} N^{\prime}=1 \bmod N_{r} ; n, k=0,1, \ldots, N-1$ are the original transform indexes; and $n^{\prime}=0,1, \ldots, N^{\prime}-1$ and $n_{r}$, $k_{r}=0,1, \ldots, N_{r}-1$ are the multidimensional indexes.

It is seen that one modulo addition is required to map each value of $n$ and $k$ in every stage of short-length transform. Note however that for a given $n^{\prime}$, the sequence $\left\{S_{2}\left(n^{\prime}, k_{r}\right)\right\}$ is just a permutation of $\left\{S_{1}\left(n^{\prime}, n_{r}\right)\right\}$. If we can establish a relation between these two sequences, we need to compute only one of them and obtain the other by a simple mapping. From eqn. 30, we have

$$
\begin{aligned}
S_{2}\left(n^{\prime}, k_{r}\right) & =\left\langle N_{r} n^{\prime}+N^{\prime} N^{\prime-1} k_{r}\right\rangle_{N} \\
& =\left\langle N_{r} n^{\prime}+N^{\prime}\left(N^{\prime-1} k_{r}\right)\right\rangle_{N} \\
& =S_{1}\left(n^{\prime}, N^{\prime-1} k_{r}\right)
\end{aligned}
$$

As both $n_{r}$ and $k_{r}$ are defined modulo $N_{r}$, the sequence $\left\{S_{2}\left(n^{\prime}, k_{r}\right)\right\}$ can simply be obtained from the sequence $\left\{S_{1}\left(n^{\prime}, n_{r}\right)\right\}$ by the mapping

$$
n_{r}=N^{\prime-1} k_{r} \bmod N_{r}
$$

Furthermore, the above mapping is independent of $n^{\prime}$, hence only one set of values is required to be evaluated for every $N_{r}$. This reduces much of the computation overheads required for the index mapping, since the evaluations of eqn. 30 are eliminated.

Consider an example with $N=3 \times 4 \times 5$ and $N_{r}=5$ : in this case $N^{\prime}=3 \times 4=12$ and $N^{\prime-1}=3$. When $n^{\prime}=3$, the sequence $\left\{S_{1}\left(n^{\prime}, n_{r}\right)\right\}$ is $\{15,27,39,51,3\}$. The permutation sequence is given by $\left\{k_{r} \bmod 5: k_{r}=0,1,2,3,4\right\}$, which is $\{0,3,1,4,2\}$. Hence the following mapping scheme should be used:

$$
\begin{aligned}
& S_{1}(3,0) \rightarrow S_{2}(3,0) \\
& S_{1}(3,3) \rightarrow S_{2}(3,1)
\end{aligned}
$$

etc.

This gives $\left\{S_{2}\left(3, k_{r}\right)\right\}$ as $\{15,51,27,3,39\}$, which is the same pattern that is generated from eqn. 30 . Note again that the permutation sequence is fixed for all $n^{\prime}$ and is required to be generated only once for each $N_{r}$.
The highly efficient modules of length-2 and 4 DHTs enable the RDHT-DFTs to be realised with a reduced number of operations. The special properties described in Section 4 further improve their efficiencies. Table 1 lists the number of real multiplications and the number of real

\begin{tabular}{|c|c|c|c|c|}
\hline \multirow[t]{2}{*}{$\begin{array}{l}\text { Transform } \\
\text { Length }\end{array}$} & \multicolumn{2}{|c|}{$\begin{array}{l}\text { Short length } \\
\text { modules for WFTA }\end{array}$} & \multicolumn{2}{|c|}{ RDHT-DFT } \\
\hline & $A$ & $M$ & $A$ & $M$ \\
\hline $\begin{array}{r}2 \\
3 \\
5 \\
7 \\
9\end{array}$ & $\begin{array}{c}2.0 \\
4.0 \\
6.8 \\
10.29 \\
9.78\end{array}$ & $\begin{array}{l}2.0 \\
2.0 \\
2.4 \\
2.57 \\
2.44 \\
\end{array}$ & $\begin{array}{l}2.0 \\
6.67 \\
11.2 \\
15.43 \\
23.64\end{array}$ & $\begin{array}{l}0.0 \\
2.0 \\
2.8 \\
6.86 \\
- \\
6.18\end{array}$ \\
\hline
\end{tabular}
additions required for some important short-length modules. To compare with the WFTA, we also include the number of operations required by its short-length modules in the same table. Table 2 further compares RDHT-DFT with several other popular algorithms,

Table 1 : Number of operations required for short length modules of WFTA and RDHT-DFT

note: $\boldsymbol{A}=$ number of real additions per point; $M=$ number of real multiplications per point

Table 2: Comparison of several fast algorithms when transform length is about 1000

\begin{tabular}{lllr}
\hline $\begin{array}{l}\text { Fast } \\
\text { algorithm }\end{array}$ & $\begin{array}{l}\text { Transform } \\
\text { length }\end{array}$ & $A$ & $M$ \\
\hline Radix-2 FFT & 1024 & 30.0 & 20.0 \\
Radix-4 FFT & 1024 & 26.0 & 12.0 \\
PFA & 1008 & 29.31 & 5.76 \\
WFTA & 1008 & 34.64 & 3.48 \\
RDHT-DFT & 1155 & 56.93 & 17.84 \\
\hline
\end{tabular}

note: $A=$ number of real additions per point; $M=$ number of real multiplications per point

namely the WFTA, the prime factor algorithm, the radix-2 FFT and the radix-4 FFT, at transform length of about 1000 . It can be observed that the present approach does not achieve minimum number of arithmetic operations. However, the number of arithmetic operations is not the only criterion in efficient realisation of DFT. Overheads like data movements and address generations often significantly affect the speed of computation, especially in high-level language implementations. In particular, popular algorithms like WFTA and FFT required large sets of multiplication constants that are not practical to be precomputed and stored as program parameters. A fair comparison should also take into consideration the time consumed for their evaluations. Appendix 10 gives a program listing of the present approach with the improved prime factor mapping described above. Fig. 2 shows a comparison of its computation speeds to those of the radix-2 FFT [11] and the WFTA [11]. All programs were written in Fortran 77 and have been executed on an IBM PC/AT-compatible machine equipped with an 80287 Math coprocessor. Note that the RDHT-DFT is of about the same speed as the WFTA, whereas the FFT is significantly slower than these two algorithms.

On the other hand, the RDHT-DFT algorithm has a further advantage of having simple and regular control structures. This simplifies the programming effort in software implementations. The program code length is 
greatly reduced by recursively using just two basic modules. This is especially beneficial in highly compositelength transforms in which several short-length transform

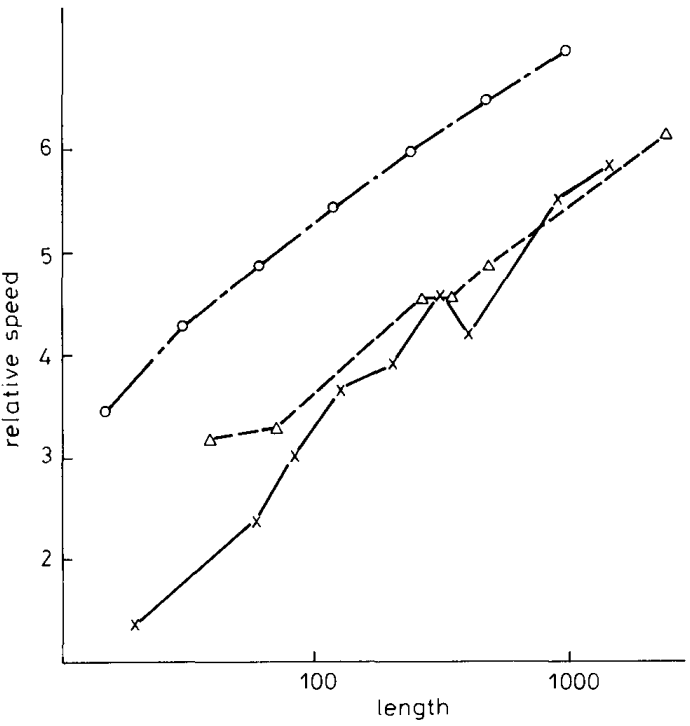

Fig. 2 Computation time per point against sequence length for DFTs - - Radix-2 FFT

- - - WFTA

RDHT-DFT

modules can share the same set of routines. These can be easily observed by comparing the program listing in Appendix 10 for the RDHT-DFT algorithm, which supports DFTs of sequence lengths composed of relatively prime factors chosen from the set $\{2,3,4,5,6,7,10,11\}$, to those found in Reference 11 for WFTA. In fact, shortlength algorithms based on previous approaches are so tedious that transform modules of length longer than 9 , except 16 , are not commonly available from literature. Hence, the RDHT-DFT algorithm is most suitable for applications which required the optimisation of programming efficiency, code length and runtime.

\section{Conclusion}

It is seen that the present approach recursively makes use of just two basic modules, namely length- 2 and length- 4 discrete Hartley transforms, and an improved prime- factor mapping to compute discrete Fourier transforms. This new approach optimises programming efficiency, code length and speed of the computation. The speed of computation is shown to be faster than the FFT and is comparable to the speed of computation for WFTA. The present algorithm has a major advantage of short program length and has a better control structure. This approach is particularly attractive in applications where program memory is very restrictive, for example in the cases of using single-chip digital signal processors. Furthermore, we would also expect to have great improvement in efficiency when the input sequence is real. The highly regular control structure of the algorithm simplifies the design of parallel computation structures for high-speed realisations.

\section{Acknowledgment}

The authors would like to acknowledge the financial support of the Research Committee of the Hong Kong Polytechnic under the project grant 340/321. K.L. Wong would also like to thank the Sir Edward Youde Memorial Fund Council for kindly awarding the research fellowship.

\section{References}

1 COOLEY, J.W., and TUKEY, J.W.: 'An algorithm for the machine calculation of complex Fourier series', Math. Comput., 1965, 19, pp. 297-301

2 GOOD, I.J.: 'The relationship between two Fourier transforms', IEEE Trans., 1971, C-20, pp. 310-317

3 WINOGRAD, S.: 'On computing the discrete Fourier transform', Math. Comput., 1978, 32, pp. 175-199

4 KOLBA, D.P., and PARKS, T.W.: 'A prime factor FFT algorithm using high-speed convolution', IEEE Trans., 1977, ASSP-25, pp. 91-103

5 RADER, C.M.: 'Discrete Fourier transforms when the number of data samples is prime', IEEE Proc., 1968, 56, pp. 1107-1108

6 SIU, W.C., and CONSTANTINIDES, A.G.: 'Very fast discrete Fourier transform, using number theoretic transform, IEE Proc. G, Electron. Circuits \& Syst., 1983, 130, (5), pp. 201-204

7 SIU, W.C.: 'A nesting algorithm for very fast discrete Fourier transform'. Proc. ICASSP '88, Apr. 1988, pp. 1898-1901

8 BRACEWELL, R.N.: 'The Hartley Transform' (Oxford University Press, 1986)

9 SORENSEN, H.V., JONES, D.L., BURRUS, C.S., and HEIDEMAN, M.T.: 'On computing the discrete Hartley transform', IEEE Trans., 1985, ASSP-33, pp. 1231-1985

10 ARAMBEPOLA, B.: 'Discrete Fourier transform processor based on the prime-factor algorithm', IEE Proc. G, Electron. Circuits \& Syst., 1983, 130, pp. 138-144

11 'Programs for digital signal processing' (IEEE Press, 1979)

\section{Appendix}

\section{COMPUTE DFT BY RDHT}

WRITTEN BY: K.L. WONG, and Dr. W.C. SIU

DEPT. OF ELECTRONIC ENGINEERING, HONG KONG POLYTECHNIC

PARAMETER PASSED:

ICODE - INDICATE THE TRANSFORM LENGTH SELECTED

DRE - THE REAL DATA SEQUENCE DIM - THE IMAGINARY DATA SEQUENCE

SUBROUTINE DHTDFT(ICODE,RSEQ,ISEQ)

REAL RSEQ(0:1539),ISEQ( $0: 1539), \mathrm{DRE}(0: 10), \mathrm{DIM}(0: 10)$

INTEGER NF(22),LEN(22),FAC(4,22),INV(4,22),MAP(0:10),PBUFF(0:10)

DATA LEN $/ 12,15,20,30,35,42,60,70,84,105,132,165,210,231,330$,

$1385,420,660,770,924,1155,1540 /$

DATA NF $/ 2,2,2,2,2,2,3,2,3,3,3,3,3,3,3,3,4,4,3,4,4,4 /$

DATA FAC $/ 3,4,0,0,3,5,0,0,4,5,0,0,5,6,0,0,5,7,0,0,6,7,0,0,3,4,5$, 
$10,7,10,0,0,3,4,7,0,3,5,7,0,3,4,11,0,3,5,11,0,5,6,7,0,3,7,11,0,5$,

$26,11,0,5,7,11,0,3,4,5,7,3,4,5,11,7,10,11,0,3,4,7,11,3,5,7,11,4$,

3 5,7,11/

DATA INV $/ 1,3,0,0,2,2,0,0,1,4,0,0,1,1,0,0,3,3,0,0,5,6,0,0,2,3,3$,

$10,5,9,0,0,1,1,3,0,2,1,1,0,2,1,1,0,1,2,3,0,3,1,4,0,2,3,10,0,1,5$,

$27,0,3,6,6,0,2,1,4,2,1,1,3,9,3,9,3,0,2,3,6,8,1,1,2,2,1,2,5,7 /$

LENGTH $=$ LEN(ICODE)

DO $500 \mathrm{I}=1, \mathrm{NF}(\mathrm{ICODE})$

ISHORT $=$ FAC(I,ICODE $)$

INVERSE $=$ INV(I,ICODE)

$\mathrm{M}=\mathrm{LENGTH} / \mathrm{ISHORT}$

$\mathrm{N}=$ ISHORT -1

IBASE $=0$

$\operatorname{MAP}(0)=0$

C ESTABLISH RELATION BETWEEN FORWARD AND INVERSE MAPPING

DO $300 \mathrm{~J}=1, \mathrm{~N}$

$\operatorname{MAP}(\mathrm{J})=\operatorname{MOD}((\operatorname{MAP}(\mathrm{J}-1)+$ INVERSE $)$, ISHORT $)$

300 CONTINUE

C FORWARD MAPPING

DO $500 \mathrm{~J}=0,(\mathrm{M}-1)$

IPTR $=$ IBASE

DO $305 \mathrm{~K}=0, \mathrm{~N}$

PBUFF(K) = IPTR

DRE $(K)=$ RSEQ(IPTR)

$\operatorname{DIM}(\mathrm{K})=\operatorname{ISEQ}(\mathrm{IPTR})$

IPTR $=$ MOD(IPTR + M,LENGTH $)$

305 CONTINUE

C APPLY CORRESPONDING DHT ON REAL AND IMAG PARTS SEPARATELY GO TO $(310,320,330,340,350,360,370,310,310,400,410)$, ISHORT

310 WRITE $(*, *)^{\prime}$ ERROR: INVALID TRANSFORM LENGTH $\rightarrow$ ',LENGTH STOP

320 CALL DHT2(DRE(0),DRE(1))

CALL DHT2(DIM(0),DIM(1))

GO TO 435

330 CALL DHT3(DRE(0),DRE(1),DRE(2))

CALL DHT3(DIM(0),DIM(1),DIM(2))

GO TO 420

340 CALL DFT4(DRE,DIM)

GO TO 435

350 CALL DHT5(DRE(0),DRE(1),DRE(2),DRE(3),DRE(4))

CALL DHT5(DIM(0),DIM(1),DIM(2),DIM(3),DIM(4))

GO TO 420

360 CALL PFA6(DRE(0),DRE(1),DRE(2),DRE(3),DRE(4),DRE(5))

CALL PFA6(DIM(0),DIM(1),DIM(2),DIM(3),DIM(4),DIM(5)) GO TO 420

370 CALL DHT7(DRE)

CALL DHT7(DIM)

GO TO 420

400 CALL PFA1O(DRE(0),DRE(1),DRE(2),DRE(3),DRE(4),DRE(5),

1 DRE(6),DRE(7),DRE(8),DRE(9))

CALL PFA1O(DIM(0),DIM(1),DIM(2),DIM(3),DIM(4),DIM(5),

1 DIM(6),DIM(7),DIM(8),DIM(9)

CALL DHT11(DRE)

CALL DHT11(DIM)

C CONVERT DHT TO DFT

420 DO $430 \mathrm{IP}=1,(\mathrm{~N} / 2)$

$\mathrm{IR}=\mathrm{ISHORT}-\mathrm{IP}$

$\mathrm{T} 1=\mathrm{DRE}(\mathrm{IP})+\mathrm{DRE}(\mathrm{IR})$

T2 $=$ DRE(IP) - DRE(IR)

$\mathrm{T} 3=\mathrm{DIM}(\mathrm{IP})+\mathrm{DIM}(\mathrm{IR})$

T4 = DIM(IP) - DIM(IR)

$\mathrm{DRE}(\mathrm{IP})=\mathrm{T} 1+\mathrm{T} 4$

$\mathrm{DRE}(\mathrm{IR})=\mathrm{T} 1-\mathrm{T} 4$

$\operatorname{DIM}(\mathrm{IP})=\mathrm{T} 3-\mathrm{T} 2$

$\operatorname{DIM}(\mathrm{IR})=\mathrm{T} 3+\mathrm{T} 2$

430 CONTINUE

C INVERSE MAPPING

IEE PROCEEDINGS, Vol. I36, Pt. E, No. 4, JULY 1989 
440 $\operatorname{ISEQ}(\operatorname{PBUFF}(\operatorname{MAP}(\mathrm{K})))=\operatorname{DIM}(\mathrm{K})$

500 CONTINUE

IBASE = IBASE + ISHORT

END

C THIS SUBROUTINE PERFORM LENGTH-2 DHT SUBROUTINE DHT2(X0,X1)

TEMP $=\mathrm{X} 0$

$\mathrm{X} 0=\mathrm{X} 0+\mathrm{X} 1$

$\mathrm{X} 1=\mathrm{TEMP}-\mathrm{X} 1$

END

C THIS SUBROUTINE PERFORM LENGTH-3 DHT SUBROUTINE DHT3(X0,X1,X2)

PARAMETER $(\mathrm{W} 1=-0.25, \mathrm{~W} 2=0.4330127)$

TEMP $=\mathrm{X} 0 * 0.5$

CALL DHT2(X2,X1)

$\mathrm{X} 0=\mathrm{X} 0+\mathrm{X} 2$

$\mathrm{X} 2=\mathrm{X} 2 * \mathrm{~W} 1+\mathrm{TEMP}$

$\mathrm{X} 1=\mathrm{X} 1 * \mathrm{~W} 2$

CALL DHT2(X2,X1)

END

C THIS SUBROUTINE PERFORM LENGTH-4 DHT

SUBROUTINE DHT4(X0,X1,X2,X3)

$\mathrm{TEMP} 0=\mathrm{X} 0+\mathrm{X} 2$

TEMP1 $=\mathrm{X} 0-\mathrm{X} 2$

$\mathrm{TEMP} 2=\mathrm{X} 1+\mathrm{X} 3$

TEMP3 $=\mathrm{X} 1-\mathrm{X} 3$

$\mathrm{X} 0=\mathrm{TEMP} 0+\mathrm{TEMP} 2$

$\mathrm{X} 1=$ TEMP1 + TEMP3

$\mathrm{X} 2=$ TEMP0 - TEMP2

$\mathrm{X} 3=$ TEMP1 - TEMP3

END

C THIS SUBROUTINE PERFORM LENGTH-5 DHT

SUBROUTINE DHT5(X0,X1,X2,X3,X4)

PARAMETER (WE4 $=-0.125, \mathrm{WE} 1=0.279508, \mathrm{WE} 3=-0.237764, \mathrm{WO} 3=-0.146946$ )

TEMP $1=\mathrm{X} 0 * 0.5$

CALL DHT4 4 X3,X4,X2,X1)

$\mathrm{X} 0=\mathrm{X} 0+\mathrm{X} 3$

TEMP2 $=\mathrm{X} 1$

$\mathrm{X} 3=(\mathrm{X} 3 * \mathrm{WE} 4)+\mathrm{TEMP} 1$

$\mathrm{X} 1=(\mathrm{X} 4 * \mathrm{WE} 3)+(\mathrm{X} 1 * \mathrm{WO} 3)$

$\mathrm{X} 2=\mathrm{X} 2 * \mathrm{WE} 1$

$\mathrm{X} 4=(\mathrm{TEMP} 2 * \mathrm{WE} 3)-(\mathrm{X} 4 * \mathrm{WO} 3)$

CALL DHT4(X3,X4,X2,X1)

END

C THIS SUBROUTINE PERFORM LENGTH-6 PFA DHT

SUBROUTINE PFA6(X0,X1,X2,X3,X4,X5)

CALL DHT2(X0,X3)

CALL DHT2(X2,X5)

CALL DHT2(X4,X1)

CALL DHT3(X0,X2,X4)

CALL DHT3(X3,X5,X1)

END

C THIS SUBROUTINE PERFORM LENGTH-7 DHT

SUBROUTINE DHT7(X)

REAL X(0:6)

PARAMETER $($ WE4 $=-0.166667$, WE3 $=0.440959$, WE5 $=-0.874842)$

PARAMETER (WO5 $=-0.111484$, WE $1=-0.734302$, WO $1=-0.488445$ )

TEMP1 $=\mathrm{X}(0)$

CALL PFA6(X(5),X(4),X(6),X(2),X(3),X(1))

$\mathrm{X}(0)=\mathrm{X}(0)+\mathrm{X}(5)$

TEMP2 $=X(4)$

TEMP3 $=\mathrm{X}(6)$

$\mathrm{X}(5)=(\mathrm{X}(5) * \mathrm{WE} 4)+$ TEMP1

$\mathrm{X}(4)=(\mathrm{X}(1) * \mathrm{WE} 5)+(\mathrm{X}(4) * \mathrm{WO} 5)$

$\mathrm{X}(6)=(\mathrm{X}(3) * \mathrm{WE} 1)+(\mathrm{X}(6) * \mathrm{WO} 1)$ 


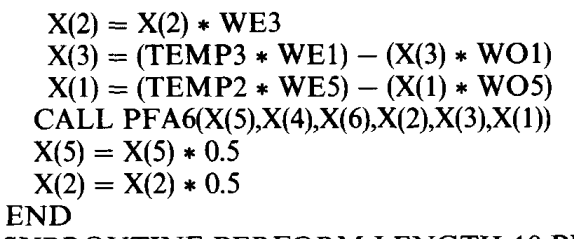

C THIS SUBROUTINE PERFORM LENGTH-10 PFA DHT SUBROUTINE PFA10(X0,X1,X2,X3,X4,X5,X6,X7,X8,X9)

CALL DHT2(X0,X5)

CALL DHT2(X2,X7)

CALL DHT2(X4,X9)

CALL DHT2(X6,X1)

CALL DHT2(X8,X3)

CALL DHT5(X0,X2,X4,X6,X8)

CALL DHT5(X5,X7,X9,X1,X3) END

C THIS SUBROUTINE PERFORM LENGTH-11 DHT

SUBROUTINE DHT11(X)

REAL X(0:10)

PARAMETER $($ WE3 $=-0.1$, WE8 $=0.331662$, WE6 $=0.0145825$, WO6 $=0.663165)$

PARAMETER (WE1 $=-0.663138$, WO1 $=-0.0157683, \mathrm{WE} 2=0.643576$ )

PARAMETER $($ WO2 $=-0.160653$, WE4 $=0.620822$, WO4 $=-0.233622$ )

TEMP1 $=\mathbf{X}(0)$

CALL PFA10(X(6),X(3),X(7),X(9),X(10),X(5),X(8),X(4),X(2),X(1))

$\mathrm{X}(0)=\mathrm{X}(0)+\mathrm{X}(6)$

TEMP2 $=X(9)$

TEMP3 $=\mathbf{X}(8)$

TEMP4 $=\mathrm{X}(1)$

TEMP5 $=X(7)$

$\mathbf{X}(6)=(\mathbf{X}(6) * \mathrm{WE} 3)+\mathrm{TEMP} 1$

$\mathrm{X}(9)=(\mathrm{X}(4) * \mathrm{WE} 6)+(\mathrm{X}(9) * \mathrm{WO} 6)$

$\mathrm{X}(8)=(\mathrm{X}(10) * \mathrm{WE} 1)+(\mathrm{X}(8) * \mathrm{WO} 1)$

$\mathrm{X}(1)=(\mathrm{X}(3) * \mathrm{WE} 2)+(\mathrm{X}(1) * \mathrm{WO} 2)$

$\mathrm{X}(7)=(\mathrm{X}(2) * \mathrm{WE} 4)+(\mathrm{X}(7) * \mathrm{WO} 4)$

$\mathrm{X}(5)=\mathrm{X}(5) * \mathrm{WE} 8$

$\mathrm{X}(2)=($ TEMP5 $*$ WE4 $)-(\mathrm{X}(2) *$ WO4 $)$

$\mathrm{X}(3)=($ TEMP4 $*$ WE2 $)-(\mathrm{X}(3) *$ WO2 $)$

$\mathrm{X}(10)=($ TEMP3 $*$ WE1 $)-(\mathrm{X}(10) *$ WO1 $)$

$\mathrm{X}(4)=($ TEMP $2 *$ WE6 $)-(\mathrm{X}(4) *$ WO6 $)$

CALL PFA10(X(6),X(3),X(7),X(9),X(10),X(5),X(8),X(4),X(2),X(1))

$\mathrm{X}(6)=\mathrm{X}(6) * 0.5$

$\mathrm{X}(5)=\mathrm{X}(5) * 0.5$

END

C THIS SUBROUTINE PERFORM LENGTH-4 DFT

SUBROUTINE DFT4(DRE,DIM)

REAL DRE(0:3),DIM(0:3)

TEMPRO $=\operatorname{DRE}(0)+\operatorname{DRE}(2)$

TEMPR $1=\operatorname{DRE}(0)-\operatorname{DRE}(2)$

TEMPR2 $=$ DRE $(1)+\operatorname{DRE}(3)$

TEMPR3 = DRE(1) - DRE(3)

TEMPI0 $=\operatorname{DIM}(0)+\operatorname{DIM}(2)$

TEMPI $1=\operatorname{DIM}(0)-\operatorname{DIM}(2)$

TEMPI2 $=\operatorname{DIM}(1)+\operatorname{DIM}(3)$

TEMPI3 = DIM(1) $-\operatorname{DIM}(3)$

DRE $(0)=$ TEMPR0 + TEMPR2

DRE $(2)=$ TEMPR $0-$ TEMPR2

DRE $(1)=$ TEMPR $1+$ TEMPI3

DRE $(3)=$ TEMPR $1-$ TEMPI 3

$\operatorname{DIM}(0)=$ TEMPI0 + TEMPI 2

DIM(2) = TEMPI0 - TEMPI2

$\operatorname{DIM}(1)=$ TEMPI $1-$ TEMPR3

END

$\operatorname{DIM}(3)=$ TEMPI $1+$ TEMPR3 